\title{
De Brumaire à la formation de l'État
} bureaucratique consulaire : le rôle des républicains conservateurs

From Brumaire to the Creation of the Bureaucratic State under the Consulate: the role of Conservative Republicans

\section{Soulef Ayad-Bergounioux}

\section{(2) OpenEdition \\ Journals}

\section{Édition électronique}

URL : https://journals.openedition.org/ahrf/13360

DOI : 10.4000/ahrf.13360

ISSN : $1952-403 X$

\section{Éditeur :}

Armand Colin, Société des études robespierristes

\section{Édition imprimée}

Date de publication : 1 décembre 2014

Pagination : 51-72

ISBN : 978-2-200-92928-2

ISSN : 0003-4436

\section{Référence électronique}

Soulef Ayad-Bergounioux, « De Brumaire à la formation de l'État bureaucratique consulaire : le rôle des républicains conservateurs ", Annales historiques de la Révolution française [En ligne], 378 | octobredécembre 2014, mis en ligne le 01 décembre 2014, consulté le 01 juillet 2021. URL : http:// journals.openedition.org/ahrf/13360 ; DOI : https://doi.org/10.4000/ahrf.13360 


\title{
DE BRUMAIRE À LA FORMATION \\ DE L'ÉTAT BUREAUCRATIQUE CONSULAIRE : LE RÔLE DES RÉPUBLICAINS CONSERVATEURS
}

\author{
Soulef AYAD-BERGOUNIOUX
}

\begin{abstract}
À compter de 1797, les républicains conservateurs, rassemblés autour de Sieyès et Rœderer, s'engagent dans le coup de force brumairien. Occupant les hautes charges administratives d'État, sous le Consulat, ils participent activement à la construction de l'État bureaucratique consulaire. Cet article se propose d'en rendre compte en s'appuyant sur un corpus d'archives inédit et sur les progrès historiographiques récents ${ }^{1}$. Ils reposent sur une « théorie matérialiste du symbolique ${ }^{2}$, qui considère que l'histoire est doublement présente : dans les structures objectives, celles de l'État consulaire, et dans la subjectivité des agents sociaux, leurs idées, leurs discours, et jusque dans leurs travaux théoriques.
\end{abstract}

Mots-clés : Consulat, État bureaucratique consulaire, Conseil d'État, républicains conservateurs.

À compter du second Directoire, une dizaine de républicains conservateurs, parmi lesquels les auteurs les plus prolifiques de la période révolutionnaire et les membres les plus actifs de l'élite administrative consulaire, ceux qui ont le plus nettement contribué à la formation de l'État bureaucratique, se rassemblent autour de Sieyès (1748-1836) et Rœderer (1754-1835). Tous s'engagent activement dans un projet commun de renversement de

(1) Voir par exemple Jean-Pierre JESSENNE (dir.), Du Directoire au Consulat : Brumaire dans l'histoire du lien politique et de l'État-Nation, Lille-Rouen, GRHIS, 2000.

(2) Pierre BOURDIEU, Sur l'État. Cours au Collège de France (1989-1992), Paris, Seuil, 2012, p. 153. 
la République directoriale. Autoproclamés « républicains modérés », en opposition aux « démagogues et royalistes », leurs objectifs sont connus et autorisent à les qualifier de républicains conservateurs. Ils entendent renverser le Directoire et instaurer une République conservatrice, reposant sur «l'égalité des droits dans l'inégalité des conditions » ${ }^{3}$. Cet article entend préciser le rôle de ces membres de l'élite « modérée » dans le coup de force brumairien sans par ailleurs dénier celui qu'ont pu y jouer d'autres forces politiques et/ou intellectuelles comme, par exemple, les « Idéologues ». La découverte récente d'une partie des archives du Conseil d'État consulaire ${ }^{4}$ - dont la grande majorité avait pourtant été détruite sous la Commune -, en a permis une connaissance plus approfondie, que cet article se propose d'exposer.

Parmi les républicains conservateurs les plus actifs, il faut compter trois présidents de sections (Intérieur, Législative et Finances) du Conseil d'État consulaire, les principaux rédacteurs de la Constitution de l'an VIII et du Code civil, et un ministre de la Justice, soit Félix Bigot Préameneu (1747-1825), Antoine Boulay de la Meurthe (1761-1840), Michel Regnaud de Saint-Jean-d'Angély (1762-1819) et Claude-Ambroise Régnier (1746-1814). Tous appartiennent à la bourgeoisie robine, c'est-àdire à ces lignées de juristes qui, après un siècle d'investissement lignager, se voient brutalement écartées du cursus honorum robin, dans les années 1780. À la suite de la crise de reproduction qui affecte la société absolutiste, tous s'engagent dans le processus révolutionnaire pour s'en éloigner ensuite ouvertement, à compter de la Terreur. Ils s'estiment alors spoliés d'un pouvoir qu'ils ont arraché de haute lutte, et qu'ils considèrent comme leur revenant de droit. Sous le Directoire, ils risquent un coup de force, en brumaire an VIII. Il s'agit de pallier l'instabilité de la période directoriale, afin de pérenniser leur position. C'est à cette fin que Sieyès et Rœderer se rapprochent d'Antoine Boulay, de Félix Bigot Préameneu, de Michel Regnaud et de Régnier. Les cinq derniers seront rejoints sous le Consulat par Joseph Bonaparte (1768-1844) .

(3) Pierre-Louis REDERER, De la philosophie moderne, et de la part qu'elle a eue à la Révolution française, Paris, Imprimerie du Journal de Paris, frimaire an VIII, p. 6-7.

(4) La reconstitution des archives du Conseil d'État a été effectuée à partir des fonds privés : Bigot de Préameneu, Gérando (Regnaud de Saint-Jean-d'Angély) et Rœderer. Voir Soulef AYADBERGOUNIOUX, Bourgeoisie de Robe et esprit d'État : genèse sociale et historique de la domination symbolique institutionnalisée (1775-1815), Thèse de Doctorat d'histoire de l'université de Paris I, soutenue le 9 juin 2012, sous la direction de M. le Professeur Pierre Serna.

(5) Voir Archives Nationales (AN.), 29 AP 12, Archives privées du comte Pierre-Louis Rœderer, Correspondances. 
Aussi la défection de l'abbé, en 1800, est-elle concomitante du processus de structuration de la nouvelle organisation étatique, née de la société révolutionnée ${ }^{6}:$ l'État bureaucratique consulaire. De fait la période consulaire marque-t-elle un tournant dans le processus révolutionnaire. La République de l'an VIII correspond à l'épisode ultime de la lutte exacerbée qui oppose, depuis dix ans, les élites, pour la mainmise sur les principaux monopoles étatiques (cœrcition physique et symbolique, juridico-administratif et fiscal). C'est, en effet, au terme des événements brumairiens que les républicains conservateurs prennent provisoirement le dessus sur leurs opposants, « démagogues et royalistes », et s'imposent comme nouvelle élite légitime, c'est-à-dire comme bourgeoisie d'État ${ }^{7}$. Les archives du Conseil d'État, notamment celles contenues dans les fonds privés Rœderer $^{8}$, et celles du pouvoir exécutif des périodes consulaire et impériale ${ }^{9}$, permettent de revenir sur l'étape finale du conflit révolutionnaire et, par là, de préciser le rôle joué par ces républicains conservateurs dans le coup de force brumairien. Enfin, leur contribution à la construction de l'État bureaucratique, en favorisant une étude précise des critères de sélection de ses administrateurs, permet de dégager la logique du mode de reproduction bureaucratique consulaire et, par là, de spécifier les fondements de la «domination bourgeoise », elle-même produit du processus révolutionnaire.

\section{Conditions historiques de possibilité d'un rapprochement des républicains conservateurs (1797-Brumaire an VIII)}

Qui sont les républicains conservateurs rassemblés autour de Sieyès et Rœderer ? Pourquoi se qualifient-ils de « républicains modérés »? À quelle date et à quelles fins politiques se sont-ils regroupés ? Autant de questions légitimes auxquelles il s'agit de répondre en recourant aux

(6) Voir Jacques BERNET, Jean-Pierre JESSENNE, Hervé LEUWERS, « Avant-propos : acquis et perspectives », dans Jean-Pierre JESSENNE (dir.), Du Directoire au Consulat : Brumaire dans l'histoire du lien politique et de l'État-Nation, op.cit., p. 20 : «nous avons voulu mettre l'accent sur ce moment spécifique [18 et 19 brumaire] du basculement d'un régime à l'autre, [...], d'autant plus quand ce moment est censé clore une révolution. Il s'agit de saisir cet accéléré où se mêlent les changements profonds, les mises en scène politiques, les falsifications des résultats du régime précédent, ce passage où se nouent les reniements d'héritage et les fidélités revendiquée. Ce moment constitue évidemment une séquence particulièrement propice à l'analyse de l'alchimie de la légitimité et des liens politiques ».

(7) Voir Archives Nationales (AN), 29 AP 18, Archives privées du comte Pierre-Louis Rœderer, Pièces relatives au 18 Brumaire et au Consulat.

(8) AN, 29 AP 79, Archives privées de Pierre-Louis Rœderer, Politique, administration, gouvernement. Manuscrits et imprimés sur la création du Conseil d'État.

(9) Ibidem, AF IV 1042, Archives du pouvoir exécutif des périodes consulaire et impériale, Fonctionnement du Ministère de la Justice : correspondances, ordres de travail, bulletins des lois. 
écrits privés (correspondances, journaux intimes) de la bourgeoisie d'État consulaire, et aux archives du Conseil d'État ${ }^{10}$.

À compter du second Directoire, les allusions à un coup de force pour rétablir « l'ordre républicain » sont légion dans les correspondances de Rœderer et de Boulay. Aussi est-il possible de supposer que l'objet premier du rassemblement d'une partie des élites conservatrices autour de Sieyès et Rœderer a pour objet le renversement de la République directoriale ${ }^{11}$. D'ailleurs les échanges épistolaires que Roderer entretient avec Boulay à l'automne 1797 le confirment. C'est sur la recommandation du second que le général Hoche (1765-1797) ${ }^{12}$ est pressenti pour devenir l'épée du prochain coup d'État. Sur les conseils de Boulay, le Mosellan s'ouvre sur les projets politiques des modérés au général Hoche :

« Je crois que les bons citoyens ont tous des reproches à faire à tous les partis. Je crois que le Directoire aura contribué à nous rendre le royalisme par son défaut d'art dans le gouvernement intérieur, autant que par son défaut de fermeté. Je crois ainsi que le royalisme nous aura rendu la Terreur par les sots [...]. Maintenant il me reste à désigner celui des généraux à qui pourra demeurer l'avantage, s'il y a une guerre civile, qui soit assez fort contre ses ennemis, contre ses amis, et contre lui-même, pour ne faire de sa victoire qu' un usage favorable à la liberté $»^{13}$.

Plusieurs éléments situent le projet de coup de force entre germinal et fructidor an V. Roderer fait allusion à la victoire des royalistes aux élections de germinal an V, et à ses conséquences politiques. Le Mosellan les déplore d'autant plus qu'elles contrarient leur projet. Ainsi, l'intention de recourir à la force constitutionnelle afin d'assurer définitivement la prééminence des "modérés » remonte-t-elle à 1797. Le rassemblement de ces républicains conservateurs le précède de peu, comme le laissent supposer les écrits épistolaires de Røderer. À cette période, les allusions

(10) Voir A.N., 29 AP 79, Archives privées de Pierre-Louis Rœderer, Politique, administration, gouvernement, Manuscrits et imprimés sur la création du Conseil d'État ; A.N. AF IV 1042, Archives du pouvoir exécutif des périodes consulaire et impériale, Fonctionnement du Ministère de la Justice : correspondances, ordres de travail, bulletins des lois; A.N. AF IV 1238, Archives du pouvoir exécutif des périodes consulaire et impériale, Attributions des conseillers d'État et Conseil d'administration de l'Intérieur sur des matières diverses.

(11) Voir AN, 29 AP 18, Archives privées de Pierre-Louis Rœderer, Manuscrits sur les 18 et 19 brumaire an VIII et AN, 29 AP 10-13, Archives privées de Pierre-Louis Rœderer, Correspondances.

(12) C'est sous le commandement de Hoche qu'Antoine Boulay de la Meurthe, devenu capitaine, participe aux exactions militaires, à la frontière du Wissembourg, en 1793.

(13) AN, 29 AP 11, Archives privées de Pierre-Louis Rœderer, Correspondance, Lettre de Roderer à Hoche datée du 13 fructidor an V(30 août 1797). 
aux « élites modérées [celles qui] s'opposent [...] courageusement aux ennemis de la Patrie [« royalistes et démagogues »] $»^{14}$ y sont d'ailleurs légion.

L'instabilité chronique du second Directoire facilite en effet le rapprochement tactique et affectif des robins libéraux et des conservateurs. La proximité idéologique qui pousse les élites «modérées » à vouloir le retour à l'ordre, c'est-à-dire la naturalisation de la hiérarchie sociale issue de la Révolution française, justifie leur rapprochement et réactive les alliances qui prévalaient en 1789. Regnaud et Rœderer, qui se connaissent pourtant depuis 1789, puisqu'ils ont tous deux été élus représentants du tiers état aux États généraux et ont fréquenté un temps les feuillants, n'approfondissent leur relation qu'à compter de la République directoriale. Leurs deux fils, Auguste Regnaud et Antoine-Marie Rœderer, élèves à Polytechnique, entretiennent également des liens étroits, et seront personnellement impliqués dans le coup de force brumairien ${ }^{15}$. Les liens qu'entretiennent les républicains conservateurs sont, pour certains, anciens. Ils sont parfois attestés avant même la Révolution. Il en va ainsi de Boulay et de Régnier. C'est en effet au second, alors avocat, qu'il revient de présenter son futur collègue à l'audience publique qui officialise sa nouvelle fonction ${ }^{16}$. Il en va de même concernant Rœderer et Régnier, qui intègrent le Parlement de Metz dans le dernier tiers du XVIII ${ }^{\mathrm{e}}$ siècle. L'ancienneté des relations que Sieyès nourrit avec ces cinq agents facilite dès lors leur rapprochement. C'est l'abbé qui introduit Bigot Préameneu au club de Valois, et le met dans le secret des événements brumairiens. Le Directoire, qui marque un véritable tournant dans la carrière politique de Boulay, est suivi d'un élargissement de son réseau. Il intègre en thermidor an $\mathrm{V}$ la commission chargée par le Conseil des Cinq-Cents d'examiner la question de «l'organisation des réunions publiques, suite à la création du Cercle Constitutionnel pour contrer les Clichyens ». C'est à cette période qu'il se rapproche des membres du parti « modéré » tels que Roederer et Sieyès. Ces derniers entretiennent une relation épistolaire intense dès 1788 . Peu après les événements thermidoriens, Rœderer se confie à l'abbé :

«malgré ma servitude privée, je souhaite, mon cher ami, que vous soyez bientôt aussi libre que moi ; que vous puissiez aussi regarder la

(14) Ibidem, 29 AP 11, Archives privées du comte Pierre-Louis Rœderer, Correspondances avec Adrien Lezay.

(15) Voir AN, 29 AP 18, Archives privées de Pierre-Louis Rœderer. Manuscrits sur les 18 et 19 brumaire an VIII.

(16) AD Meurthe-et-Moselle, 3 B XXI 7. 
Seine couler comme je le fais et vais le faire plus que jamais de mes fenêtres ; enfin grommeler ensemble sur l'espèce humaine [...] et réaliser enfin à nous deux la grande faction des insociables [...]. Je vous embrasse tendrement $»^{17}$.

Le ton amical de la lettre et l'ancienneté de leur relation ne font pas de doute. Enfin Regnaud et Sieyès se rapprochent dès l'été 1791, où tous deux adhèrent aux Feuillants. Regnaud n'est pas étranger au rapprochement de l'abbé et du général, qu'il fréquente assidûment à compter de l'an III.

Malgré la proximité idéologique et affective des « modérés », il leur a fallu plus de deux ans pour mettre en œuvre le projet de renversement de la République directoriale. C'est que les événements fructidoriens, les décès consécutifs des généraux Hoche et Joubert les ont contraints à porter leur dévolu sur Bonaparte, dont ils se défiaient unanimement. Les écrits épistolaires de Rœderer sont sans équivoque. Il correspond à plusieurs reprises avec Boulay, notamment à propos du plan constitutionnel de l'an VIII :

«Craignant de ne pas vous trouver, je vous dis ici, mon cher Rœderer, qu'ayant parlé hier au citoyen Sieyès de la disposition où vous étiez de travailler à la rédaction des articles constitutionnels, il en a été enchanté. Voyez le donc le plus tôt possible, il vous remettra tous ses matériaux à cet égard. Je vous salue, Boulay ${ }^{18}$.

En comparant les informations contenues dans ce billet que Boulay destine à Rœderer, à celles divulguées par le journaliste dans sa Notice, il est possible de préciser la nature des relations qui lient Bonaparte aux républicains « modérés » et par là même, de préciser le rôle de chacun dans le coup de force :

«Talleyrand et moi [Rœderer] fûmes les deux intermédiaires qui négocièrent entre Sieyès et Bonaparte. Tous les yeux étaient ouverts sur l'un ou sur l'autre. Nous nous étions interdit toute entrevue particulière [...]. Talleyrand était l'intermédiaire qui concertait les démarches à faire et la conduite à tenir. Je fus chargé de négocier les conditions politiques d'un arrangement $»^{19}$.

(17) Ibidem, 29 AP 109, Archives privées de Pierre-Louis Rœderer, Littérature, philosophie, morale. Lettre de Roederer à Sieyès, datée du 12 août 1795.

(18) Ibid., 29 AP 10, Archives privées de Pierre-Louis Rœderer, Correspondances. Billet de Boulay à Roederer, non daté.

(19) Ibid., 29 AP 8, Archives privées de Pierre-Louis Rœderer, Divers (XVII -XVIII ${ }^{e}$ siècles). 
Regnaud occupe alors des fonctions semblables à celles de Talleyrand, mais cette fois pour le compte de Bonaparte. Apparemment Boulay et Rœderer sont responsables, respectivement, pour Sieyès et Bonaparte, de la gestion de leurs relations politiques. Pourtant tous deux sont intimement liés à Sieyès. La correspondance que Rœderer entretient avec « son ami » Sieyès est éloquente. Aussi est-il probable qu'il s'agisse ici d'une manœuvre de ces républicains conservateurs, qui s'inquiètent des conséquences d'un coup d'État dans lequel un général est personnellement engagé. Les écrits épistolaires de Røderer le confirment :

«On me dit, mon cher ami, que vous refusez la place qui vous est conférée. Je vous supplie, je vous conjure au nom de la patrie et de la liberté de prendre encore quelques jours pour interroger votre conscience sur ce que vous êtes en droit de faire. Ne nous abandonnez donc pas à la perversité et à la stupidité $»^{20}$.

En exhortant Sieyès à faire fi du différend politique qui l'oppose à Bonaparte sur la question du pouvoir exécutif, Rœderer dévoile la prédominance de sa relation avec Sieyès sur celle qu'il entretient avec Bonaparte. Il en va de même pour Boulay qui regrette, dans ses Mémoires, les soupçons injustifiés de Sieyès à son égard :

«Le soir du même jour, j'allai chez Sieyès ; je le trouvai sombre et son accueil fut très froid. Il était persuadé, comme je l'appris le lendemain [par Rœderer], que je l'abandonnais pour me tourner entièrement du côté de Bonaparte. Il se trompait assurément $»^{21}$.

Fidèles à la tradition rousseauiste, tous trois se méfient des conceptions de Bonaparte en matière de pouvoir exécutif. Boulay en fait état dans ses Mémoires. Sieyès s'oppose à un pouvoir exécutif trop puissant et s'en explique à Boulay : « la tendance naturelle du gouvernement [l'ordre exécutif] est d'augmenter sans cesse ses attributions et ses prérogatives $»^{22}$. C'est pourquoi, il désire le voir partagé entre deux consuls et quatorze ministres, chargés de l'exécution des lois. Surtout, le Collège des conservateurs, futur Sénat, dont la « principale destination est de maintenir la constitution dans toute sa pureté. [Il peut ostraciser] [...] tout citoyen éminent par ses talents et ses services [pouvant] exercer sur la multitude un ascendant

(20) Ibid., 29 AP 12, Archives privées de Pierre-Louis Rœderer, Correspondances. Billet de Rocerer à Sieyès, daté de brumaire an VIII.

(21) Antoine BOULAI DE LA MEURTHE, Mémoires, op.cit., p. 52-53.

(22) Ibidem, op.cit., p. 22. 
[...] général et aveugle $»^{23}$ et ainsi troubler l'ordre public. Pour Sieyès, le pouvoir exécutif doit trouver son équilibre dans les contre-pouvoirs établis en son sein. Conscient des risques liés à la personnalisation du pouvoir, il s'applique à lui trouver un palliatif. Or le général ne l'entend pas ainsi. L'absence d'alternative amène Boulay comme Rœderer à céder sur cet aspect. Ainsi, la proximité politique et affective de ces agents avec Sieyès supplante celle qu'ils ont développée avec Bonaparte. Aussi le coup d'État brumairien est-il, pour une bonne part, le fait des républicains « modérés », notamment Sieyès, Rœderer, Boulay, Regnaud, attachés à justifier leur pouvoir et ainsi à s'imposer comme élite légitime. Au soir du 19 Brumaire, il leur reste à justifier leur coup de force, à en effacer l'arbitraire. Ils ont non seulement joué un rôle central dans la préparation et l'exécution des événements brumairiens, mais ils sont en outre placés au cœur du processus de légitimation du coup de force de novembre 1799.

C'est par un coup de force légal que les républicains conservateurs, désireux de rompre le compromis constitutionnel de l'an III, s'imposent comme nouvelle élite dominante. Brumaire ne suscite pourtant aucune révolte locale ${ }^{24}$. Comment expliquer ce relatif consensus ${ }^{25}$ ? L'analyse des discours, placards et articles de presse produits par les conjurés durant la phase de légitimation du nouveau pouvoir, soit entre les mois de brumaire et frimaire an VIII permet d'y répondre. Afin de légitimer leur pouvoir, ils adoptent au soir du 19 Brumaire une double stratégie. Ils envoient dans les municipalités des missionnaires chargés du maintien de l'ordre public et justifient, par des discours officiels prononcés au sein des anciennes chambres représentatives directoriales, leur coup de force. L'absence de soulèvement témoigne de leur efficacité. Aussi leurs discours peuvent-ils être considérés comme une méta-sociodicée, c'est-à-dire une justification théorique par les dominants de leur nouveau pouvoir, dont la légitimité est renforcée par l'officialisation qui en efface l'arbitraire. Ainsi, Boulay, président de la commission chargée « d'examiner la cause de ses maux [la République directoriale] et d'indiquer les moyens de les faire cesser », argue de la légalité et de la nécessité du coup de force de l'an VIII

(23) Ibid, p. 32-42.

(24) Voir notamment le chapitre 9, «Du Directoire au Consulat : un laboratoire pour une idéologie de l'extrême centre », dans Pierre SERNA, La République des Girouettes, Paris, Champ Vallon, 2005 p. 414-466 ; Isser WOLOCH, Napoleon and his collaborators, The making of a dictatorship, New York, London, Norton, Norton, 2001.

(25) Maurice AgulHon, La vie sociale en Provence intérieure au lendemain de la Révolution, Paris, Société des Études robespierristes, 1970, p. 405 : «Tout se passe donc comme si la chute du Directoire, interprétée par les notables comme une promesse d'ordre, l'avait été par les masses populaires comme l'annonce d'un retour à la vie normale ». 
afin de le justifier. D'après lui, l'alliance avec les « néo-jacobins » apparaît désormais bien inutile, d'autant qu'elle se serait avérée néfaste pour les «modérés ». Les conditions de l'an II ne sont pas celles de l'hiver 1799. À lui seul, le compromis constitutionnel de l'an III expliquerait l'instabilité de la République. Regnaud et Rœderer, rédacteurs des adresses aux Français placardées dans tout Paris, le 19 Brumaire, recourent à d'identiques arguments pour justifier le coup de force : faire face aux menaces que font peser les "véritables ennemis de l'Intérieur », " démagogues et royalistes », sur la République, en les excluant du jeu politique ; réformer une république « agonisante », reposant sur un compromis de circonstance, celui de thermidor an II. Il est temps maintenant d'établir les bases d'un « ordre social juste» assis sur les principes de propriété, de liberté et d'égalité des droits dans l'inégalité des conditions ${ }^{26}$. Tout comme Boulay, ils réactivent les pratiques discursives de l'an III, renvoyant dos-à-dos " démagogues et royalistes » ${ }^{27}$, et invoquent le contexte extérieur troublé de l'été 1799, pour justifier le recours à des mesures extrêmes. L'effet structurant de la tempérance dans les pratiques discursives des hommes de loi est dès lors peu surprenant. Elle est comme consubstantielle à une profession qui dit le droit après avoir procédé à l'examen impartial, dépassionné des faits. Le modérantisme seul peut assurer la paix d'une société gouvernée par ces hommes maîtres d'eux-mêmes et capables, par suite, de discernement. C'est pourquoi les «modérés » rapprochent continuellement la situation de l'été 1792 de celle de Brumaire. À l'été 1799 un emprunt forcé de cent millions est levé sur les riches. La loi sur les otages est adoptée le 24 messidor an VII, soit le 12 juillet 1799. Trois jours après, un toast à « la résurrection des piques » est publiquement porté par le général Jourdan. Effrayés, les «modérés » ferment le Manège, club politique où se regroupent les « républicains démocrates ». Le contexte est propice à la réactivation des schèmes cognitifs stigmatisants de l'élite juridique bourgeoise, et dont est pleine la sociodicée, qui justifie ses privilèges. Cette dernière relègue les classes populaires à une position inférieure et les rapproche, en symétrie inversée, des « royalistes » et des « démagogues », dont elles partageraient les tares, notamment la haine du travail et du talent: "Dans l'intérieur, des ennemis presque aussi

(26) AN, 29 AP 18, Archives privées de Pierre-Louis Rœderer, Pièces concernant les 18 et 19 Brumaire. Machiavel.

(27) Voir Marc DELEPLACE, «Tensions discursives et mises en cohérence du discours sur l'anarchie (1793-1799 », dans Marc DELEPLACE, L'Anarchie de Mably à Proudhon (1750-1850). Histoire d'une appropriation polémique, Paris, ENS éditions, 2001, p. 115-145. 
redoutables que l'étranger, se sont plusieurs fois relevés au milieu de nos alarmes ; la sanglante anarchie a plusieurs fois offert le combat à la basse tyrannie, et c'est à leurs débats qu'a tenu jusqu'à présent le salut des citoyens. Cependant quels accommodements n'a pas obtenu l'anarchie? Des impôts spoliateurs ont ébranlé la propriété ; la loi des otages a détruit la sûreté personnelle ; la guerre civile s'est allumée et embrase une partie de la République ; une portion nombreuse de l'armée des Français est poussée au désespoir ; la circulation des subsistances est arrêtée $»^{28}$. Les républicains conservateurs incriminent ouvertement les «néo-jacobins » qui ont tenté de résister au coup de force. C'est pourquoi, les « séditieux, les furieux », bref les «anarchistes » sont rendus responsables de la situation. Mais une place est ménagée pour les élites révolutionnaires et royalistes qui se soumettraient au nouvel ordre des choses : « si l'on considère, par exemple, le Conseil d'État une année après Brumaire, on dirait que Fructidor n'a jamais eu lieu. Assis les uns à côté des autres, on retrouve plusieurs victimes des purges ou déportations de Fructidor (des hommes comme Benézech, Portalis, Emmery, Fleurieu) et plusieurs partisans du coup de Fructidor (Boulay de la Meurthe, Berlier, Réal, Français de Nantes) $»^{29}$. Cette double logique d'intégration et d'exclusion des « anarchistes et des royalistes » suffit à légitimer le coup d'État. C'est que le dénigrement des « ennemis de l'Intérieur », « néo-jacobins et royalistes », la peur sociale qu'inspirent les revendications politiques populaires sont des poncifs désormais largement répandus dans la société consulaire. L'action de la presse libérale et conservatrice - Le Conservateur, La Décade, le Journal de Paris -, qui s'évertue depuis l'an V à les publiciser, a certes grandement favorisé l'adhésion à de tels préceptes ${ }^{30}$. Mais c'est essentiellement parce qu'il existe un consensus sur les valeurs, le modérantisme et l'attachement à la propriété, que les citoyens acceptent aussi facilement le coup de force. C'est pourquoi celui-ci a pu constituer l'étape finale du conflit ouvert dès 1789 entre élites révolutionnaires et contre-révolutionnaires.

À l'hiver 1799-1800, ce groupe de républicains conservateurs et leurs alliés finissent par provisoirement l'emporter. Ils s'imposent alors comme bourgeoisie d'État et engagent leur conception du monde social

(28) Michel Regnaud DE SAINT-JEAn D’AngÉly et Pierre-Louis ROEDERER, Un Français aux Français, dans AN, 29 AP 18, Archives privées de Pierre-Louis Rœderer, Pièces concernant les 18 et 19 Brumaire an VIII.

(29) Isser WOLOCH « Les dynamiques locales d'un coup d'État », dans Jean-Pierre JESSENNE (dir.), Du Directoire au Consulat : Brumaire dans l'histoire du lien politique et de l'État-Nation, op.cit., p. 131-132.

(30) Voir Pierre SERNA, op.cit., p. 446-447. 
et leur système de valeurs dans la construction de la nouvelle structure étatique. Leur compétence juridique les rend indispensables à l'organisation du nouvel ordre consulaire. C'est parce que la bourgeoisie d'État parvient à s'imposer comme élite légitime, à la suite des événements brumairiens, qu'elle a désormais la possibilité de délimiter le cadre au sein duquel les agents luttent pour le monopole du capital étatique.

\section{Le champ bureaucratique consulaire ou la lutte pour le monopole des instruments de pouvoir}

L'État bureaucratique consulaire est une invention de la Robe révolutionnée, notamment des républicains conservateurs rassemblés, à compter de l'hiver 1799-1800, autour de Rœderer et de Joseph Bonaparte. En tant que fictio juris, il est forgé par l'élite juridique bourgeoise, qui y investit son ethos, c'est-à-dire un système de valeurs. C'est en le considérant comme tel qu'il sera dès lors possible de déconstruire les mécanismes du système de reproduction bureaucratique, et par là, de préciser le processus d'institutionnalisation, donc de légitimation, du pouvoir de l'élite administrative d'État de la période consulaire. Le champ bureaucratique, en tant que subjectivité institutionnalisée, reproduit les structures cognitives de l'ethos modéré, auxquelles il ajoute une légitimité propre que lui confère son inscription dans l'ordre des lois. L'analyse du fonctionnement interne du Conseil d'État, et plus particulièrement l'étude des critères de sélection des conseillers d'État, permet de saisir les mécanismes cachés, invisibles, sur lesquels repose le maintien de l'ordre social, c'est-à-dire la reconnaissance par le plus grand nombre du bien-fondé de la hiérarchie sociale issue de la société révolutionnée, qui situe la Robe à son sommet. À cette fin seront préalablement définies les fonctions des conseillers d'État, dont Boulay et Rœderer, sont justement les inventeurs. Il est institué par arrêté consulaire du 4 nivôse an VIII (25 décembre 1799). Trente-quatre conseillers d'État sont nommés (article I). Ils sont répartis en cinq sections : Guerre, Marine, Finances, Législation, Intérieur. Celles-ci sont chargées sous le Consulat, de l'organisation puis de la gestion des principaux monopoles étatiques (juridique, économique, coercition physique et symbolique). Les sections de l'Intérieur et de la Législation sont les plus importantes ${ }^{31}$. Elles ont en charge l'organisation administrative

(31) Voir Marie-Catherine VIGNAL, Les imprimés du Conseil d'État de l'époque napoléonienne, Paris, Mémoire de D.E.A, 1994. 
et judiciaire de la République, autrement dit celle du monopole juridicoadministratif. Ce dernier, en tant que monopole symbolique sublimé, permet au groupe qui le détient d'assurer, par l'objectivation, la légitimité de son pouvoir. Sous la République consulaire, les républicains « modérés » en sont les principaux détenteurs. En effet, en l'an IX (1800-1801), Boulay et Rœderer sont les présidents des principales sections du Conseil d'État, Intérieur et Législative. C'est ce que laisse transparaître le règlement du 25 décembre 1799, qui organise et définit les fonctions législatives du Conseil d'État. Il regroupe les principaux administrateurs de la République, auxquels il confie l'essentiel des prérogatives en matière législative.

La Constitution de l'an VIII ainsi que le Règlement du 4 nivôse an VIII, dont les notes se trouvent dans les archives du Conseil d'État, permettent de préciser les fonctions des conseillers d'État. Quelles sont leurs attributions constitutionnelles ? En vertu de l'article 52 de la Constitution du 22 frimaire an VIII, le Conseil d'État, sous la direction des consuls, est chargé de rédiger les projets de loi et les règlements administratifs, ainsi que de résoudre les problèmes afférents à ces questions (article XI). Dans les faits, il a l'initiative des lois. Sous la République consulaire, la formation des lois nécessite trois démarches distinctes, qui sont autant de limites officielles assignées aux pouvoirs exécutif et législatif, que se partagent les consuls, notamment le Premier d'entre eux, Bonaparte, et les conseillers d'État : la proposition et la rédaction des lois, la discussion et leur proclamation. Les conseillers d'État ont en charge les deux premières étapes. Ils transmettent ensuite leur projet aux ministres. Ces derniers, à leur tour, le soumettent aux consuls (article VII). Les lois sont ensuite discutées au sein de la section concernée du Conseil d'État. Les consuls y sont présents. Les ministres également, mais leur avis est uniquement consultatif (articles VIII, IX et XI). En vertu de l'article IX de l'arrêté du 4 nivôse an VIII, après accord des consuls, les règlements sont adoptés sans discussion préalable devant le Tribunat. Ce dernier discute uniquement les lois. Aussi les ministres ont-ils été écartés de la rédaction des actes constitutionnels. Dans leur grande majorité, les réformes constitutionnelles et administratives ont été adoptées par règlement. C'est le cas du Conseil d'État. La principale chambre représentative n'intervient qu'en aval. Ce sont les conseillers d'État qui disposent de l'initiative des lois, en accord avec les consuls qui sont chargés de la nomination du conseiller d'État auquel revient la rédaction de la loi. L'accord du Tribunat est néanmoins nécessaire à l'adoption à cette dernière. En cas de contentieux, la loi est à nouveau discutée au sein du Corps législatif, qui décide ou non de la valider. En dernier recours, ce sont les conseillers d'État qui, à la demande 
des consuls, statuent sur ladite loi. Ils sont également chargés de la gestion des litiges sur « le sens des lois » (article XI). Lorsqu' un ministre estime qu'un changement est nécessaire, il doit présenter un avis motivé aux consuls. Ces derniers le renvoient à l'examen de la section compétente. Les demandes des ministres sont alors discutées au sein de la section du Conseil concernée. Celle-ci motive son avis auprès du consul qui, à son tour, la renvoie au Conseil d'État. C'est à ce dernier que revient en définitive la décision de tenir compte de l'avis des ministres. Il est jugé en fonction de sa conformité avec les principes de la Constitution, les vues du gouvernement et l'intérêt de l'État, eux-mêmes préalablement définis par la bourgeoisie d'État. En définitive, sous la République consulaire, les conseillers d'État ont l'initiative des lois et des règlements, les consuls ne faisant que valider un état de fait. Les décisions prises en amont par les juristes sont majoritairement validées. Ainsi l'élite juridique bourgeoise est la fondatrice de l'État bureaucratique consulaire. C'est elle qui détient le monopole du capital étatique, à son profit.

Les archives du Conseil d'État le confirment. Les fonds privés Rœderer contiennent l'un des rares procès-verbaux d'une des séances de la section de l'Intérieur du Conseil d'État, au moment où elle remplit les fonctions d'assemblée constituante ${ }^{32}$. Il porte sur le débat qui a précédé l'instauration des listes de notabilités, instituées par la Constitution de l'an VIII. Il prouve que, dans les faits, le pouvoir de décision des consuls et notamment du Premier Consul, Napoléon Bonaparte, est limité par sa relative exclusion de l'administration bureaucratique. Bonaparte est catégoriquement opposé à l'instauration de listes de notabilités. Durant cette séance, il ne cesse d'interpeller les conseillers d'État sur la faisabilité d'un tel projet : « La Constitution permet-elle de former des assemblées électorales et de déléguer ainsi le droit d'élire $»^{33}$ ? Plusieurs des républicains « modérés » (Boulay, Régnier, Regnaud) interviennent et se rangent apparemment du côté de Napoléon Bonaparte. Ils prolongent son questionnement, mais en tirent des conclusions opposées. En prétextant du caractère anticonstitutionnel des listes de notabilités, Bonaparte envisage de limiter le pouvoir des élites administratives d'État, ses principaux opposants, qui, en déterminant les listes des élus à l'échelle locale, pourraient contrecarrer son influence. Regnaud et Régnier, après avoir feint de partager les inquiétudes de Bonaparte, n'hésitent pas à prendre un parti contraire :

(32) AN, 29 AP 76, Archives privées de Pierre-Louis Rœderer. Sur la représentation politique, Procès verbal de la séance du 18 thermidor an VIII (6 août 1800), portant sur les listes de notabilités.

(33) Ibidem. 
«Les citoyens Regnier et Regnaud [...] ne regardent pas le texte de la Constitution comme assez impératif pour qu'on ne puisse, si on le croit convenable, recourir à la formation d'assemblées électorales $»^{34}$. [Rœderer surenchérit] : «Le citoyen Rœderer [...] croit contraire à l'esprit de la constitution et aux principes de la liberté, tout mode de désignation qui forcerait les votants de sacrifier le juste désir d'aller chercher plus loin un Citoyen probe et capable, à l'obligation de choisir autour d'eux $»^{35}$.

Or ce sont les conseillers d'État qui finissent, sous le Consulat, par l'emporter. «Les articles 7,8 et 9 de la Constitution ordonnent la formation de listes graduelles, où seront inscrits les citoyens jugés les plus propres à gérer les affaires publiques dans les magistratures communales, départementales et nationales. L'article 14 veut que ces listes soient formées, pour la première fois, dans le cours de l'an $9 »^{36}$. Les listes de notables sont dressées à partir d'un seul et même critère, « le mérite de ceux qui se sont distingués par leur service à l'État ${ }^{37}$. Elles seront ensuite ratifiées par le peuple tous les trois ans, et les sortants pourront être reconduits. Ainsi, malgré l'article 41 de la Constitution, qui donne le droit au Premier Consul Bonaparte de nommer les ministres, les ambassadeurs, les officiers, les magistrats et les membres des administrations locales, les conseillers d'État finissent par imposer leur conception du monde social. En imposant les listes de notabilités, ils prescrivent leur conception de l'administrateur idéal, donc leur ethos. C'est en fonction de leur système de valeurs qu'est déterminée la position des administrateurs d'État dans le champ bureaucratique. Le pouvoir de Bonaparte est limité par le cadre même de ces nominations. C'est au sein des listes de notabilités, qu'il est contraint de choisir les fonctionnaires. Or, ces listes sont établies à la discrétion du président de la section de l'Intérieur du Conseil d'État et du ministre de l'Instruction publique, fonctions respectivement occupées par Rœderer entre 1799 et 1802, et entre mars et septembre 1802. En prescrivant les principes d'ordonnancement du champ administratif, la bourgeoisie d'État impose ses normes politiques comme dominantes. C'est parce qu'elle dispose au lendemain de Brumaire, de la mainmise sur le capital juridique, sorte de capital symbolique sublimé, qu'elle parvient

(34) Ibid.

(35) Ibid.

(36) Pierre-Louis ROEDERER, Motifs du projet de loi concernant la formation et le renouvellement des listes d'éligibilité prescrites par la constitution, Paris, Imprimerie nationale, an VIII, p.1-2.

(37) Ibidem. 
à imposer sa propre définition du conseiller d'État. Elle s'assure ainsi le monopole des hautes charges administratives d'État. Les critères de sélection des auditeurs du Conseil d'État le confirment.

C'est par un arrêté du 19 germinal an $\mathrm{XI}^{38}$ que sont créés les auditeurs du Conseil d'État dont Régnier est le fondateur. Ils sont chargés de préparer les dossiers que devra examiner le Conseil. Ils constituent la première étape de la carrière administrative d'État :

« un auditeur [...] n'est pas comme le serait un fonctionnaire subalterne dans une administration centrale, voué à des tâches obscures en attendant que son âge lui permette de faire mieux. Il est directement au contact des hauts dignitaires, et souvent même chargé de missions ou de fonctions importantes $»^{39}$.

Leur nombre est initialement arrêté à seize. Mais dès 1804, ils sont soixante-douze puis deux cent cinquante-neuf, en 1813. À compter de décembre 1809, un décret stipule que pour être nommé auditeur, il faut être âgé d'au moins vingt ans, avoir rempli ses obligations militaires et disposer d'au moins six mille francs de revenus annuels. En outre, à partir de janvier 1813, l'obtention d'une licence en droit ou de la licence ès sciences est une obligation légale ${ }^{40}$. Ce resserrement dans la sélection des auditeurs ne fait qu'entériner un état de fait. C'est ce que montrent les archives du pouvoir exécutif ${ }^{41}$. Le mérite associé au travail est, au même titre que la recommandation et l'aisance financière, un critère essentiel à la sélection des candidats. Les propriétés distinctives de la bourgeoisie d'État (l'éloquence civique, le désintéressement, le modérantisme, le mérite associé au talent, le labeur) sont transfigurées par la reconnaissance officielle qui leur est accordée. On les retrouve dans les Observations concernant les candidats :

«il a reçu une bonne éducation; c'est un jeune homme de vingt ans qui a fait d'excellentes études ; il est très instruit ; animé de talent ; annonce d'excellentes dispositions ; beaucoup de zèle au travail ; élève de l'école polytechnique, sujet distingué ; homme studieux et d'une excellente

(38) AN, AF IV 1042, Archives du pouvoir exécutif des périodes consulaire et impériale, Fonctionnement du Ministère de la Justice : correspondances, ordres de travail, bulletins des lois.

(39) Christophe CHARLE, Les Hauts fonctionnaires en France au XIX ${ }^{e}$ siècle, Paris, Gallimard, p. 206

(40) Voir également Jean TULARD, « Le Conseil d'État de Napoléon », dans Roland DRAGO, Jean IMBERT, Jean TULARD, François MONNIER, Dictionnaire biographique des membres du Conseil d'Etat, 1799-2002, Montrouge, Fayard, 2004, p. 13-16.

(41) AN, AF IV 1042, Archives du pouvoir exécutif des périodes consulaire et impériale, Fonctionnement du Ministère de la Justice : correspondances, ordres de travail, bulletins des lois. 
conduite ; il a développé de l'activité et des talents ; il a du zèle et des connaissances, $[\ldots]$; on le regarde comme un des meilleurs ; instruit, ayant du zèle et de l'activité ; bon sujet, du talent ; bon administrateur, laborieux ; probe, environné de confiance et d'estime $»^{42}$.

Comme le montrent les descriptions des auditeurs « méritant » leur intégration au Conseil d'État, c'est tout leur être social qui structure in fine l'identité de la nouvelle élite administrative. En 1809, les auditeurs au Conseil d'État sont dans leur grande majorité licenciés en droit ou es sciences. C'est le cas de $78 \%$ d'entre eux. La plupart ont fréquenté l'École Polytechnique. Tous ont été introduits par un membre de l'administration étatique. Ainsi Arnault, « fils du secrétaire général de l’Université impériale, neveu de Regnaud de Saint-Jean-d'Angély » comme «Combis Sieyès [qui] a épousé la nièce du Sénateur Sieyès $»^{43}$, sont tous deux rapidement nommés conseillers d'État. Près de $85 \%$ des auditeurs sont indépendants financièrement. Leur rente qui se monte en moyenne à 8000 francs annuels, les situe du côté de l'aisance relative ${ }^{44}$. Capacités et capitaux sont deux critères essentiels dans la sélection des auditeurs. Ils témoignent de l'alliance des élites économiques et juridiques. En institutionnalisant ses critères de nomination, la bourgeoisie d'État oblige les élites de la nation, économiques notamment, à adopter les règles de la condition robine. L'ethos modéré est ainsi transmué en système officiel de valeurs, autrement dit en dispositions permanentes, qui permet à la bourgeoisie de Robe et à ses alliés d'accaparer les hautes charges administratives d'État. Par l'officialisation de son ethos, l'élite juridique bourgeoise efface l'arbitraire de son pouvoir et pérennise sa domination. Elle impose ses schèmes classificatoires (mérite, désintéressement, modérantisme et éloquence civique) comme normes universelles de toute hiérarchie, c'est-à-dire de tout ordre social $^{45}$. Dès lors, la lutte politique se transmue en une lutte cognitive pour l'imposition de la vision acceptable et reconnue du monde social. Elle est ontologiquement liée à la réforme des structures administratives d'État.

(42) Ibidem.

(43) Ibid.

(44) Les calculs ont établi à partir des Archives du pouvoir exécutif des périodes consulaire et impériale, conservées aux archives nationales (AN, AF IV 1042).

(45) Christophe CHARLE, Les Hauts fonctionnaires [...], op.cit., p. 10-11 : « cet ouvrage ne sera qu'assez peu chronologique dans la mesure où le temps administratif n'obéit pas au rythme rapide de l'histoire événementielle mais se rapproche plutôt de la longue durée chère à F. Braudel. Il ne s'agit pas là d'une pétition de principe antihistorique mais de l'expression de la continuité sociale de la haute fonction publique, assez peu entamée par les épurations ou les démissions des personnages liés à tel ou tel régime ». 
Elle facilite la normalisation du champ révolutionnaire. Aussi, sous le Consulat et sous l'Empire, les conflits sont-ils limités au champ du pouvoir. C'est ce que laissent transparaître les conflits qui opposent les "modérés », rassemblés autour de Rœderer et Joseph Bonaparte, à Fouché, puis aux « bonapartistes », sous l'Empire.

\section{Les luttes au sein du champ administratif sous l'Empire}

À la suite de Brumaire, la normalisation du champ de luttes révolutionnaire aboutit à la structuration du champ bureaucratique, transmué en espace normé au sein duquel les élites sont en concurrence pour le monopole du capital étatique, qui donne pouvoir sur les autres formes de capital (économique, culturel, social) et permet la manipulation légitime des biens publics. Il a pour effet de circonscrire les luttes de pouvoir au champ administratif et, par là, d'en limiter l'effet déstabilisant. Le conflit opposant les « modérés » à Fouché et à Napoléon Bonaparte, est un exemple particulièrement révélateur de la stabilisation du champ révolutionnaire transformé, sous le Consulat, puis sous l'Empire, en champ bureaucratique au sein duquel les élites luttent pour le monopole légal et légitime du capital étatique « donnant pouvoir sur les différentes espèces de capital et sur leur reproduction $»^{46}$. À l'hiver 1799-1800, le contexte est favorable à l'élite administrative d'État. Ses membres parviennent à imposer leur plan constitutionnel. La défection de Sieyès, qui suit de près la proclamation de la Constitution de l'an VIII, n'entraîne pas leur chute. C'est à cette période qu'ils se regroupent autour de Joseph Bonaparte, le frère aîné de Napoléon, qui se présente alors comme un fervent défenseur de la République consulaire. Les liens qui l'unissent à Rœderer sont anciens. Ils remontent au Directoire :

«d'étroites relations s'étaient dès longtemps établies entre Joseph Bonaparte et Rœderer. Elles remontaient à l'époque où Joseph, revenu de son ambassade à Berlin, fut appelé à siéger au Conseil des Cinq Cents comme représentant de la Corse. [...]. Ils étaient unis d'opinion et se voyaient sans cesse $\gg^{47}$.

(46) Pierre BOURDIEU, Loïc J. D. WACQUANT, Réponses. Pour une anthropologie réflexive, Paris, Seuil, 1992, p. 71-90. Voir également Pierre BOURDIEU, Sur l'État, op.cit.

(47) AN, 29 AP 8, Archives privées de Pierre-Louis Rœderer, Papiers de famille. Quelques notes sur Joseph Bonaparte. 
Rœderer rassemble ses proches Boulay, Regnaud, Régnier, autour de Joseph Bonaparte. Ils seront ponctuellement rejoints par Talleyrand, Lucien Bonaparte, Félix Bigot Préameneu. Entre 1799 et 1815, les «modérés » entrent en concurrence ouverte avec les « bonapartistes » pour le monopole du capital étatique. Pourtant le conflit qui les oppose à Fouché (1754-1820) sous le Consulat éclipse rapidement celui engagé dès le commencement des événements brumairiens avec le Premier Consul. Cet épisode constitue un moment significatif et méconnu des luttes qui traversent le champ bureaucratique consulaire. Non seulement il lui est circonscrit, mais il ne s'ensuit encore aucune remise en cause de la domination de l'élite juridique bourgeoise. Cette dernière s'est imposée comme figure légitime de l'administrateur d'État en institutionnalisant son ethos. Les conflits personnels, dès lors qu'ils ne remettent pas en cause les critères de définition institutionnalisés des élites administratives d'État, se ramènent à des nonévénements, parce qu'entièrement limités à l'espace bureaucratique. C'est le cas du conflit qui oppose une partie des républicains conservateurs à Fouché et, à travers lui, aux « démagogues ».

Les diatribes des robins à l'égard de Fouché, « l'ami des terroristes », jusqu'alors restreintes à un cadre strictement privé, sont ouvertement exprimées au sein du champ bureaucratique, à partir du printemps 1802. Elles supplantent les récriminations de Boulay et Rœderer contre le Consulat à vie. Après s'être ponctuellement rapprochés de Fouché, en raison de leur opposition au Concordat, ils en viennent à soutenir le projet de Bonaparte. Leur échec, puisque le Concordat est institué en avril 1802, met fin à l'alliance de circonstance qui les rapprochait du ministre de la Police. Les liens de Joséphine Bonaparte avec Fouché achèvent de rapprocher les « modérés », pourtant méfiants à l'égard du Premier Consul, de Napoléon Bonaparte $^{48}$. Fouché éprouve des sentiments tout à fait semblables à leur égard. Il se méfie des « collaborateurs du Premier Consul [...], il ne cherche pas à les gagner, mais rassemble sur chacun d'eux des fiches $»^{49}$. Roderer multiplie les pétitions contre Fouché. Elles sont toutes signées par les

(48) Voir également Louis MADELIN, Joseph Fouché, Paris, Nouveau Monde Éditions, 2010 (1 $1^{\text {ère }}$ édition 1901), p. 307-308: « Et il a raison de craindre personnellement ces fatales conséquences [instauration du Consulat à vie]. Il les connaît bien depuis nivôse, les gens qui dirigent le nouveau gouvernement monarchique : il les déteste, ils le haïssent ; Rœderer, Lucien, Talleyrand, quelques nobles exaspérés contre le jacobin. Le Consulat à vie, c'est le triomphe de ses détracteurs, ses ennemis personnels. [...]. Contre les Lucien, les Talleyrand, les Rœderer, Fouché n'a qu'un recours : Joséphine. C'est une alliée, nous avons dit pourquoi. Elle ne veut pas du Consulat à vie ».

(49) Albert SOBOUL, «Joseph Fouché », dans Albert SOBOUL (dir.), Dictionnaire historique de la Révolution française, Paris, PUF, 1989, p. 466. 
républicains conservateurs. S'ils se sont accommodés de l'indispensable aide de Fouché, alors ministre de la Police au moment du coup de force brumairien, ils s'évertuent à limiter l'influence de cet « homme odieux », qui a participé aux «ignominies » perpétrées par les «terroristes de $1793 »^{50}$. Pour ce faire, Joseph Bonaparte, Boulay, Regnaud, Régnier, soutenus par Bigot Préameneu et Lucien Bonaparte, s'attachent en vain à discréditer Fouché, en le présentant comme un allié des "démagogues ». Non seulement il finance leur principal journal, Le Journal des Hommes libres, mais il fait fi des réserves des élites « modérées » à l'égard du régime consulaire : "Qu'a donc voulu le Ministre de la Police ? Nous faire servir de pâture à la horde féroce dont il trouve bon d'assouvir la faim $»^{51}$. Ces membres de l'élite administrative d'État nourrissent une aversion similaire pour les «démagogues », les «sauvages » et Fouché, tous étrangers à l'ethos du Haut fonctionnaire d'État, c'est-à-dire « celui-là seul [qui] doit être appelé de ce nom [de moraliste, car], connaissant la source d'où procède la morale et le but où elle doit tendre, [il] sait employer, pour faire sentir ses commandements et leur assurer l'obéissance, toutes les circonstances de la vie qui ont prise sur le cœur de l'homme et déterminent ses habitudes. Le moraliste est le législateur par excellence et l'homme d'État du premier rang $»^{52}$. Seulement le Premier Consul qui « le craint [Fouché] $»^{53}$, hésite à se débarrasser du « gêneur $»^{54}$, puisqu'il conforterait nécessairement la position des républicains conservateurs. Soucieux d'éviter un renversement du rapport de forces au profit de Bonaparte, Roederer se refuse à son tour à publiciser son opposition au Ministre de la Police. Dans une des pétitions qu'il adresse au général, il s'en explique : «j'ai cru qu'il ne convenait pas à un homme attaché au Gouvernement, surtout à un Magistrat, d'en attaquer un autre et d'offrir le scandale d'une division entre deux hommes appelés à concourir à un même but ${ }^{55}$. Sa haine à l'égard des « anarchistes » et autres traîtres est palpable :

(50) AN, AF IV 1042, Archives du pouvoir exécutif, Fonctionnement de la Justice, correspondances avec l'Empereur, ordre de travail, bulletins des lois.

(51) AN, 29 AP 6, Archives privées de Pierre-Louis Rœderer. Papiers de famille, Pétition contre Fouché, Ministre de la Police, au sujet des injures que se permet habituellement contre moi le Journal des Hommes libres.

(52) Antoine-Marie REDERER, Euvres de Pierre-Louis Roderer, publiées par son fils, Paris, Typographie de Firmin Didot frères, 1854, T. V., p. 121.

(53) Albert SOBOUL, « Joseph Fouché », dans Albert SOBOUL (dir.), art.cit., p. 466.

(54) Ibidem.

(55) AN, 29 AP 6, Archives privées de Pierre-Louis Rœderer, Papiers de famille. Pétition contre Fouché, Ministre de la Police, au sujet des injures que se permet habituellement contre moi le Journal des Hommes libres. 
«il [Fouché] a favorisé et [...] il favorisera toujours leur renaissance, sans en avoir peut-être le dessein ; il la favorisera parce que l'infamie de sa vie passée, la multiplicité et l'énormité de ses crimes révolutionnaires, la bassesse de son esprit, de son langage, de ses manières, ses habitudes, ses liaisons l'ont identifié avec la canaille révolutionnaire, parce qu'il vit constamment avec eux dans la plus indignante familiarité. Parce que des égorgeurs connus, lui prennent les mains et le tutoient, quand ils l'abordent, parce qu'ils le regardent tous comme un camarade de boucherie $»^{56}$.

Comme le laisse transparaître Rœderer à travers ce texte, lui et ses alliés (Bigot Préameneu, Boulay de la Meurthe, Regnaud de SaintJean-d'Angély, Régnier) craignent une probable déstabilisation de la République consulaire par les « néo-jacobins ». Fouché cristallise la somme des rancunes rentrées de la bourgeoisie d'État consulaire à l'égard des « démagogues » et de leurs alliés, les classes populaires. Ils en viennent à soutenir des mesures qui fragilisent leur position, comme l'instauration du Consulat à vie, mesure à laquelle Fouché le « régicide » est pourtant opposé. La logique du jeu finit par supplanter celles des intérêts initiaux des agents. Les prises de position des élites du champ du pouvoir dépendent de la position qu'elles y occupent et des alliances qu'elles sont amenées à contracter. Ainsi, c'est en cédant sur la question du Consulat à vie, contraire à leur intérêt, que les conseillers d'État obtiennent le soutien de Napoléon Bonaparte, qui finit par écarter Fouché en 1802. De leur point de vue, l'urgence est d'exclure définitivement les « traîtres » et les « anarchistes » du champ du pouvoir. Les manœuvres de ces membres de l'élite administrative d'État, emportés par leur haine à l'égard des « anarchistes », montrent la présence dans l'imaginaire robin de toute l'histoire révolutionnaire, notamment des luttes qui les ont opposés à la « bourgeoisie » radicale, à compter du printemps 1791.

La normalisation du champ révolutionnaire et ses implications, notamment l'évolution des règles de la lutte politique, sont majoritairement méconnues comme telles par les groupes concurrents. Les agents qui ont pourtant créé l'organisation bureaucratique consulaire sont dominés par un jeu dont ils ont eux-mêmes déterminé les règles. L'analyse que ces cinq républicains conservateurs font de leur déclin relatif sous l'Empire le confirme. S'ils continuent à accaparer les hautes charges administratives d'État, ils se plaignent ainsi continuellement des blessures que leur inflige le 
comportement despotique de l'Empereur. Dès septembre 1802 Rœderer est congédié du ministère de l'Instruction publique qu'il dirigeait depuis quatre mois seulement. Avec le soutien de Joseph Bonaparte, il est nommé ministre des Finances du Royaume de Naples, en 1808. Régnier est également démis de ses fonctions de ministre de la Justice en 1807 avant d'être rétabli, en 1813. Félix Bigot Préameneu est nommé ministre des Cultes, en 1808. Peu après avoir été proclamé "empereur des Français », en vertu du sénatus-consulte du 18 mai 1804, Napoléon manifeste symboliquement son pouvoir. Il rétablit Fouché dans ses prérogatives de ministre de la Police. En novembre de la même année, il fait fermer la Bibliothèque du Conseil d'État, principal lieu de sociabilité des «modérés ». Feignant l'incompréhension, Regnaud, un des membres les plus actifs de ce groupe, le « supplie d'accueillir avec bonté ses objections $»^{57}$. La Bibliothèque sera néanmoins fermée et les livres détruits. L'animosité de Napoléon à leur égard est ancienne. Elle remonte aux événements brumairiens, à la suite desquels il a été indirectement évincé du jeu politique. Niant ou prétextant, selon les circonstances, la technicité des débats juridiques précédant la formation de l'État bureaucratique consulaire, les cinq conseillers d'État en ont exclu Bonaparte et ses affidés. Bigot de Préameneu, Boulay, Regnaud, Régnier et Rœderer y ont tous participé. Le comportement extravagant de Napoléon, sous l'Empire, les réveillant en pleine nuit et les giflant ne $\mathrm{s}^{\prime}$ explique pas autrement ${ }^{58}$. Ainsi ils sont, au même titre que Napoléon, dominés par les règles du jeu politique qu'ils ont eux-mêmes élaborés. C'est leur propre conception du monde qu'ils engagent dans la construction de l'État bureaucratique. C'est celle-ci qui structure l'ordre social consulaire, et partiellement l'ordre impérial.

Le coup de force brumairien favorise l'arrivée au pouvoir de ces républicains conservateurs, autoproclamés «modérés » qui, en tant que membres du nouveau groupe dominant, exercent différemment le pouvoir. Il s'ensuit une relative stabilisation du champ révolutionnaire - espace de luttes non codifiées et par là continuellement déstabilisé par les conflits exacerbés de tous contre tous pour le monopole du pouvoir -, transmué en champ du pouvoir où le rapport de forces entre élites concurrentes est régi par des normes implicites, découlant d'une nouvelle organisation étatique élaborée par le nouveau groupe dominant et ses alliés. En accaparant le

(57) AN, AF IV 1042, Archives du pouvoir exécutif, Fonctionnement de la Justice, correspondances avec l'Empereur, ordre de travail, bulletins des lois.

(58) Voir AN, 29 AP 8, Archives privées de Pierre-Louis Rœderer, Sur Bonaparte. 
capital étatique, ces membres de l'élite administrative d'État officialisent la conception dominante des événements brumairiens et en effacent ainsi l'arbitraire. Ils institutionnalisent les schèmes classificatoires de leur groupe, la bourgeoisie d'État, au sommet desquels se situe leur ethos. Ils imposent leur conception du monde social comme légitime, et dominent en dominant l'État. Seulement, il faut souligner au terme de cet article que, s'ils sont actifs dans la formation de la nouvelle organisation étatique, c'est néanmoins l'histoire, c'est-à-dire les luttes au sein du champ du pouvoir absolutiste et du champ révolutionnaire, entre les années 1780 et 1799, qui à travers eux agit et la façonne. Aussi sont-ils doublement dominés, par le jeu politique et par la conception qu'ils s'en font. Ils s'engagent dans la lutte pour la mainmise sur le capital étatique en méconnaissant les fondements historiques de leurs prises de position. Or c'est la méconnaissance des conditions objectives de la structuration du champ bureaucratique qui explique la pérennité de la domination robine sous l'Empire. C'est pourquoi la bourgeoisie d'État s'évertue continuellement à dénier la violence des conflits internes au champ du pouvoir. La modération est contraignante pour un ethos qui la place au sommet des qualités de l'homme d'État. C'est cet ethos, ce système de valeurs, qui structure le mode de reproduction bureaucratique qui s'institutionnalise sous le Consulat et permet aux nouveaux dominants, parmi lesquels les républicains «modérés », de s'imposer comme nouvelle élite et de pérenniser ainsi leur position.

Soulef AYAD-BERGOUNIOUX Université Paris Sud - Jean Monnet. Centre Droit et Sociétés Religieuses (EA - 1611) 54, Boulevard Desgranges 92331 SCEAUX Cedex - France soulef.ayad@club-internet.fr 